\title{
NF2-Related Intravestibular Schwannomas: Long-Term Outcomes of Cochlear Implantation
}

Huan Jia 12 , Yann Nguyen 13 , Baptiste Hochet 1, Mustapha Smail 1 , Isabelle Mosnier 1 , Hao Wu 2, Olivier Sterkers 1132 , Michel Kalamarides 4 3, Daniele Bernardeschi 1

\author{
Affiliations \\ 1 APHP, GH Pitié-Salpêtrière, Department of Otology, Auditory Implants and Skull \\ Base Surgery, Ile-de-France Reference Center of Cochlear Implant and Auditory Brainstem \\ Implant. \\ 2 Department of Otolaryngology Head and Neck Surgery, Shanghai Ninth People's \\ Hospital, Shanghai Jiaotong University School of Medicine, Shanghai, China. \\ 3 Sorbonne Universités, Paris, France. \\ 4 APHP, GH Pitié-Salpêtrière, Department of Neurosurgery, Rare Diseases Center of \\ NF2.
}

\begin{abstract}
Objective: Intravestibular schwannomas (IVSs) are uncommon tumors in Neurofibromatosis type 2 (NF2) and are mainly associated with multiple internal auditory meatus (IAM) and cerebellopontine angle (CPA) tumors. They usually induce profound hearing loss which can be rehabilitated by cochlear implantation (CI). The aim of this study was to analyze the longterm outcomes of CI during the unpredictable evolution of NF2 disease.
\end{abstract}

Study Design: Retrospective case review.

Setting: Tertiary referral center.

Patients: Three adults with neurofibromatosis type 2 and intravestibular schwannomas, and who were cochlear implant recipients.

Interventions: Periodic radiologic follow-up, tumor resection and hearing rehabilitation.

Main outcome measures: Audiological evolution, tumor evolution, surgical outcome, cochlear implant outcome.

Results: Three NF2 patients (mean age at diagnosis, $21 \pm 2.5$ years) were identified with IVS in the period between 2000 and 2014. IVS were first observed by serial MRI and profound hearing loss occurred in this ear after $4 \pm 1.5$ years of follow-up. IVS were removed via a translabyrinthine (TL) approach, and ipsilateral cochlear implantations were simultaneously performed. In two patients, large contralateral CPA tumors had previously been removed without hearing preservation, whereas in the third patient, a small, growing contralateral VS was excised via a retrosigmoid approach 6 months after IVS removal/cochlear implantation with serviceable hearing preservation. In all cases, CI provided good hearing outcomes. In two cases, hearing outcomes were even better for more than 5 years when ipsilateral intracanalicular vestibular schwannomas were removed in either the same or subsequent procedures.

Conclusions: Rehabilitation of hearing with CI provides a favorable long-term outcome in patients with NF2-related IVS which could be altered by the occurrence of other intracanalicular and/or CPA NF2-related tumors. 
Keywords: neurofibromatosis type 2, vestibular schwannoma, intra-labyrinthine tumor, intravestibular tumor, cochlear implant 


\section{INTRODUCTION}

Neurofibromatosis type 2 (NF2) is a genetically determined disorder whose main manifestation is the development of benign nerve sheath tumors along the cranial nerves with 90-95\% of patients developing bilateral vestibular schwannomas (VS). This tumorigenesis can be found anywhere along the vestibular nerve from the cerebellopontine angle (CPA) to the inner ear. When these tumors arise primarily in the inner ear, they are called intralabyrinthine vestibular schwannomas (ILS) $(1,2)$. These intralabyrinthine lesions can be classified into several subtypes, including intravestibular schwannoma (IVS), intracochlear schwannoma, etc. $(3,4,5)$.

NF2-related ILS have rarely been reported despite their high prevalence in temporal bone on NF2 cadavers (6). The impossibility of surgical preservation of residual hearing while removing intralabyrinthine tumors makes surgical timing and procedure different from NF2-"classic" VS without intralabyrinthine involvement, and consequently hearing rehabilitation by cochlear implant (CI) should be counseled when hearing is lost (7-9). In this study, we present three cases of NF2 with IVS which were followed up for more than 12 years. After IVS excision, CI achieved high performance on long-term especially as intracanalicular associated-VS had also been removed through a translabyrinthine (TL) approach.

\section{PATIENTS AND METHODS}

Following institutional review board approval, the authors carried out a retrospective review of NF2 patients and the cochlear implant register in a French tertiary medical center for the period between 2000 and 2017 in order to identify patient with IVS and in whom a cochlear implantation had been performed. The age at diagnosis, sex, hearing status and evolution (AAO-HNS classification (10)), tumor size and evolution, pre-operative management, surgical records, rehabilitated hearing outcome, and imaging data were collected.

The surgical indications for these patients were discussed by a multidisciplinary team composed of the otologists, neurosurgeon, audiologist, speech therapist, neuro-radiologist and psychologist. The surgery was performed with collaboration between the senior otologists and the neurosurgeon. The intraoperative auditory tests were performed by the senior audiologist. Pure tone and speech audiometry in a quiet environment were performed in a soundproof room. CI performance was evaluated using open-set word (WRS) and sentence (SRS) recognition scores in quiet and noise (Fournier bisyllabic words and sentences lists). VS size was measured on enhanced-gadolinium magnetic resonance images (MRI) by the senior neuro-otologists, and included both the maximal CPA diameter classified in four stages (1: intracanalicular; 2: $<15 \mathrm{~mm} ; 3:<30 \mathrm{~mm} ; 4: \geq 30 \mathrm{~mm}$ ) and its internal auditory meatal (IAM) invasion (11). Postoperative MRI controls were performed when necessary by $1.5 \mathrm{~T}$ MRI under bandaging protocol if cochlear implantation had been carried out. 


\section{RESULTS}

Two male (cases 1 and 2) and one female patient (case 3) with unilateral NF2-related IVS were identified in the study period. The intralabyrinthine lesions were strictly restricted to the vestibule (Fig 1A, 2A, 3A), and these lesions were identified several years after that contralateral large VS had been excised first in cases 1 and 2. The mean age at diagnosis of IVS was 26.3 years (range: 24 - 30 years). IVS were associated with ipsilateral intracanalicular VS in 2 cases (Fig 1C and 2B) which were recognized later on, or small CPA meningioma in the last one (Fig 3A). No other NF2-related tumor was observed in these patients. Family history with a gene mutation identification was found in case 3. Observation with serial MRI and audiometric tests were initially undertaken for them, and hearing on the IVS side was lost after $4 \pm 1.5$ years without local tumor growth. All the 3 cases were implanted with cochlear implant concomitantly to IVS removal through TL. One year after cochlear implantation, WRS achieved $87 \pm 9.4 \%(n=3)$ however the evolution of NF2-related tumors differed between cases during their follow-up of $10 \pm 5.1$ years.

In case 1 (male, 30 years-old), large right VS was excised in another department through RS but hearing could not be preserved. Left IVS which was already visible at diagnosis in 2000 (Fig 1A) was recognized 3 years later as hearing deteriorated rapidly on the only hearing ear. IVS was removed and a cochlear implant (Cochlear CI 24) positioned concomitantly (Fig 1B). CI performance progressively declined over time and a $6 \mathrm{~mm}$-intracanicular VS was disclosed 8 years after implantation (Fig 1C) and appeared to grow quite rapidly after 6 months (Fig 1D). IAM decompression through MF was performed (2011) with an immediate recovery of CI performance. However, CI performance declined again 2 years later because of a fast growing VS which extended slightly into the CPA (overall length of VS, $11 \mathrm{~mm}$ ) (Fig $1 \mathrm{E})$. The tumor could be dissected from the auditory nerve and was completely removed by enlargement of the previous TL approach to expose the IAM and CPA while the CI was left in place (2013). Immediately after surgery, recovery of CI performance was noticed and the patient felt that his hearing had improved sound quality. This good performance has remained stable for the last 5 years (WRS: $90 \%$; SRS: $100 \%, 87 \%, 73 \%$ in quiet, noise $+10 \mathrm{~dB}$ SNR, and noise $+5 \mathrm{~dB}$ SNR, respectively) and was even better than after the initial IVS removal and cochlear implantation 15 years earlier (Cochlear CI24, 20 active electrodes). In the meantime, a symptomatic C6 ependymoma was excised (2015). In 2018, the patient is tumor-free (Fig $1 \mathrm{~F})$ and is working as a data processing consultant and can use phone.

For case 2 (male, 24 years-old), excision of a large VS was performed through TL in another department in 2005. Because of a left progressive and fluctuating hearing loss in the only hearing ear, MRI was performed and revealed both IVS and small nodular intracanalicular VS in 2009 (Fig 2A). The patient was referred to us in 2011 as hearing had declined such that he had no benefit of hearing aid. IAM decompression by MF did not achieve any benefit (2012) and consequently excision of both IVS and intracanalicular VS and cochlear implantation (Med-EL Concerto, $31 \mathrm{~mm}$ ) were performed through TL during the same surgical procedure 
(2013). Five years later, the patient presented bilateral small $(3 \mathrm{~mm})$ and stable enhancement of the fundus of the IAM (Fig 2D), and was at work and a phone user. CI performance (11 active electrodes) in 2018 was: WRS $76 \%$ and SRS $100 \%, 80 \%, 80 \%, 33 \%$ in quiet and in noise, $+10,+5$, and $0 \mathrm{~dB}$ SNR, respectively.

In case 3 (female, 25 years-old), a right sudden hearing loss had recovered partially under steroid therapy in 2000 and NF2-related tumors were evidenced with IVS and CPA meningioma on the right side and intracanalicular VS on the left one (Fig 3A); Because left VS was growing rapidly with a normal hearing (Fig 3B), and on the right side hearing was non-serviceable, both right IVS and CPA meningioma were removed through TL and CI implanted (Cochlear CI24) concomitantly in 2002. Then left stage 2 VS was excised through RS 6 months later (2003). Hearing was preserved in the left ear with a slight decrease of 15 $\mathrm{dB}$ (PTA) and the patient was CI user to restore binaural cues. During the first postimplantation 3 years CI performance remained high but slightly deteriorated (90\% WRS and $100 \%$ SRS at 1 year vs $60 \%$ WRS and SRS at 3 years). The patient was lost of followed-up for several years and another MRI was performed 10 years after CI implantation when CI did not achieve any benefit and had been abandoned. Meningioma recurrence on the right side was observed (Fig 3C) and removed. CI was explanted as cochlear nerve was not preserved during the surgical procedure although post-operative facial nerve function remained normal (2014). Binaural hearing was rehabilitated by CROS hearing aids. Two years later, hearing declined slightly on the left only hearing ear and another right menigioma and a possible left de novo or recurrent intracanalicular VS were found (Fig 3D). Then treatment with Bevacizumad was started recently as hearing still declined and NF2-related tumors grew.

\section{DISCUSSION}

Although the largest NF2 cadaver study to date reported the presence of ILS in $88 \%$ of ears (6), at the present time, clinical prevalence is lower at about $15 \%$ and $8 \%$ of ears in NF2 and sporadic VS $(3,12)$, respectively. In fact, cadaver studies were mainly performed on the individuals with more aggressive NF2 disease compared with the living NF2 patients. Furthermore, intracochlear schwannomas (50-51\%) occurred more frequently than intravestibular ones (19-29\%) in a systematic review (4,5), as well as in the cadaver study (6). Schwannomas strictly restricted to the vestibule are rare conditions even in NF2 as only three cases were found in our database of more than 250 NF2 patients. Progressive or fluctuating hearing loss was the main symptom although there was no extension into the cochlea. Hearing was lost in a short period of observation (4 years) without clear tumor growth, at variance with the much slower hearing decline with NF2 $(13,14)$ or with sporadic VS $(15,16)$. This may be accounted for by direct damage to the organ of Corti by local abnormal metabolites, such as elevated intralabyrinthine protein, or interruption of potassium cycling $(12,17)$.

Surgical resection of intralabyrinthine tumors with hearing preservation has been considered 
to be impossible $(4,7,18)$. Thus it has been proposed to remove these tumors when hearing was lost and to perform cochlear implantation in a single surgical procedure through a TL approach to avoid the occurrence of intracochlear fibrosis and/or ossification which might preclude a subsequent electrode array insertion. In such cases, CI provided a good outcome whether the ILS was excised from the cochlea or not $(8,19,20)$. However, long-term CI performance might be altered by either ILS recurrence or tumor development within the ipsilateral IAM. This occurred in the present study more than 5 years after cochlear implantation by either VS growth in cases 1 and 2, or meningioma recurrence in the IAM and CPA in case 3. Indeed, the decreased CI performance may be accounted for by compression of both auditory nerve and cochlear blood supply as evidenced by the transient benefit observed after IAM decompression $(20,21)$ and the improved CI performance with subsequent VS removal (case 1). Accordingly, in case 2, IAM decompression had no effect on fluctuating hearing loss which was induced by tumor development within the vestibule. However, long-term CI benefit was achieved by resection of both IVS and intracanalicular VS. On the other hand, IAM invasion by meningioma precluded preservation of the auditory nerve so the CI had to be explanted (case 3). At present time chemotherapy (bevacizumad) could be advocated to preserve CI benefit (22) and IAM restricted to CI performance decline in case of growing intracanalicular VS. Further NF2 tumorigenesis should be monitored in these young patients although the presence of the magnetic artifact in case 3 made MRI follow-up difficult. This might be overcome by an experienced neuro-radiological team or an improved positioning of the receiver/stimulator (23-25), as in case 2.

In conclusion, these three IVS cases were initially treated in the early 2000 s by surgical removal which might not be at the present time the first-line treatment for NF2 apart from cases with life-threatening large VS $(26,27)$. However, this study demonstrates that removal of IVS with concomitant CI implantation provides a long-term benefit from CI, although it could be altered by the occurrence of other intracanalicular and/or CPA NF2-related tumors, the treatment of which might restore the initial CI benefit.

\section{REFERENCES}

1. Wolf JS, Mattox DE. Imaging quiz case 2: Intralabyrinthine schwannoma. Arch Otolaryngol Head Neck Surg 1999;125:107-9.

2. Tieleman A, Casselman JW, Somers T, et al. Imaging of intravestibular schwannomas: a retrospective study of 52 cases with emphasis on lesion growth. Am J Neuroradiol 2008;29:898-905.

3. Kennedy RJ, Shelton C, Salzman KL, Davidson HC, Harnsberger HR. Intralabyrinthine schwannomas: diagnosis, management, and a new classification system. Otol Neurotol 2004 Mar;25(2):160-7.

4. Van Abel KM, Carlson ML, Link MJ, et al. Primary inner ear schwannomas: a case series and systematic review of the literature. Laryngoscope 2013 Aug;123(8):1957-66. 
5. Choudhury B, Carlson ML, Jethanamest D. Intralabyrinthine Schwannomas: Disease Presentation, Tumor Management, and Hearing Rehabilitation. J Neurol Surg B Skull Base. 2019 Apr;80(2):196-202.

6. Nam SI, Linthicum FH, Merchant SN. Temporal bone histopathology in neurofibromatosis type 2. Laryngoscope 2011;121(7):1548-54.

7. Jia H, Marzin A, Dubreuil C, et al. Intravestibular schwannomas: symptoms and managements. Auris Nasus Larynx 2008;35(1):131-6.

8. Aschendorff A, Arndt S, Laszig R, et al. Treatment and auditory rehabilitation of intravestibular schwannoma by means of cochlear implants. HNO 2017;65(Suppl 1): 46-51.

9. Plontke SK, Kösling S, Rahne T. Cochlear implantation after partial or subtotal cochleoectomy for intracochlear schwannoma removal - A technical report. Otol Neurotol 2018 Mar;39(3):365-71.

10. Monsell E, Balkany T, Gates G, et al. Committee on Hearing and Equilibrium guidelines for the evaluation of hearing preservation in acoustic neuroma (vestibular schwannoma). Otolaryngol Head Neck Surg 1995;113:179-80.

11. Zhang Z, Nguyen Y, De Seta D, et al. Surgical treatment of sporadic vestibular schwannoma in a series of 1006 patients. Acta Otorhinolaryngol Ital 2016 Oct;36(5): 408-14.Asthagiri AR, Vasquez RA, Butman JA, et al. Mechanisms of hearing loss in neurofibromatosis type 2. PLoS One 2012;7(9):e46132.

12. Asthagiri AR, Vasquez RA, Butman JA, et al. Mechanisms of hearing loss in neurofibromatosis type 2. PLoS One 2012;7(9):e46132.

13. Evans DG. Neurofibromatosis type 2 (NF2): a clinical and molecular review. Orphanet J Rare Dis 2009;4:16.

14. Peyre M, Bernardeschi D, Sterkers O, et al. Natural history of vestibular schwannomas and hearing loss in NF2 patients. Neurochirurgie. 2018 Nov;64(5):342-7.

15. Hunter JB, Dowling EM, Lohse CM, et al. Hearing outcomes in conservatively managed vestibular schwannoma patients with serviceable hearing. Otol Neurotol 2018 Sep; 39(8):e704-e711.

16. Sughrue ME, Yang I, Aranda D, et al. The natural history of untreated sporadic vestibular schwannomas: a comprehensive review of hearing outcomes. J Neurosurg 2010 Jan; 112(1):163-7.

17. Frisch CD, Eckel LJ, Lane JI, et al.. Intralabyrinthine schwannomas. Otolaryngol Clin North Am 2015 Jun;48(3):423-41.

18. Bouchetemblé P, Heathcote K, Marie JP. Intralabyrinthine schwannomas: A case series with discussion of the diagnosis and management. Otol Neurotol 2015 Feb;36(2):e64-5.

19. Bento RF, Gebrim EM, Magalhães AT, et al. Minimally invasive surgery for intracochlear schwannoma removal and simultaneous cochlear implantation. Int Arch Otorhinolaryngol 2016 Jul;20(3):271-4. 
20. Slattery WH, Hoa M, Bonne N, et al. Middle fossa decompression for hearing preservation, a review of institutional results and indications. Otol Neurotol 2011 Aug; 32(6):1017-24.

21. Bernardeschi D, Peyre M, Collin M, et al. Internal auditory canal decompression for hearing maintenance in neurofibromatosis type 2 patients. Neurosurgery 2016 Sep;79(3): $370-7$.

22. Plotkin SR, Stemmer-Rachamimov AO, Barker FG, et al. Hearing improvement after bevacizumab in patients with neurofibromatosis type 2. N Engl J Med 2009 Jul 23;361(4):358-67.

23. Tod I, Rademacher G, Mittmann P, et al. MRI artifacts and cochlear implant positioning at 3T in vivo. Otol Neurotol 2015;36:972-6.

24. Walton J, Donelly NP, Tam YC, et al. MRI without magnet removal in Neurofibromatosis type 2 patients with cochlear and auditory brainstem implants. Otol Neurotol 2014;35:821-5.

25. Shew M, Bertsch J, Camarata $\mathrm{P}$, et al. Magnetic resonance imaging in a neurofibromatosis type 2 patient with a novel MRI-compatible auditory brainstem implant. J Neurol Surg Rep 2017 Jan;78(1):e12-e14.

26. Jia H, El Sayed MME, Smail M, et al. Neurofibromatosis type 2: Hearing preservation and rehabilitation. Neurochirurgie 2018 Oct 9. pii: S0028-3770(18)30306-0. doi: 10.1016/j.neuchi.2018.09.003. [Epub ahead of print]

27. Tan H, Jia H, Li Y, et al. Impact of cochlear implantation on the management strategy of patients with neurofibromatosis type 2. Eur Arch Otorhinolaryngol 2018 Nov;275(11): 2667-74. 


\section{Figure legends:}

Figure 1. Case 1. Imaging from 2000 to 2018 showing the undiscovered IVS at diagnosis (A) which was removed with concomitant cochlear implantation (B), and then ipsilateral growing VS (C-E). No recurrence was noticed on the last follow-up (F).

Figure 2. Case 2. MRI from 2009 to 2018 showing the undiscovered IVS at diagnosis (A) and ipsilateral slow growing VS (B-C). On the last follow-up, bilateral small enhancement were observed without any evidence of recurrence (D).

Figure 3. Case 3. MRI from 2000 to 2018 showing IVS (A) associated with other NF2related tumors (ipsilateral CPA meningioma and contralateral intracanalicular VS), contralateral growing VS after 1 year (B) and ipsilateral meningioma recurrence 14 years later (C). Final imaging disclosing another CPA meningioma which may have been hidden by the magnet artifact on previous MRI and non-visible left lesion on this view (D). 

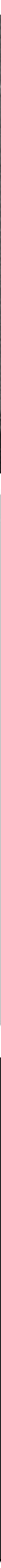

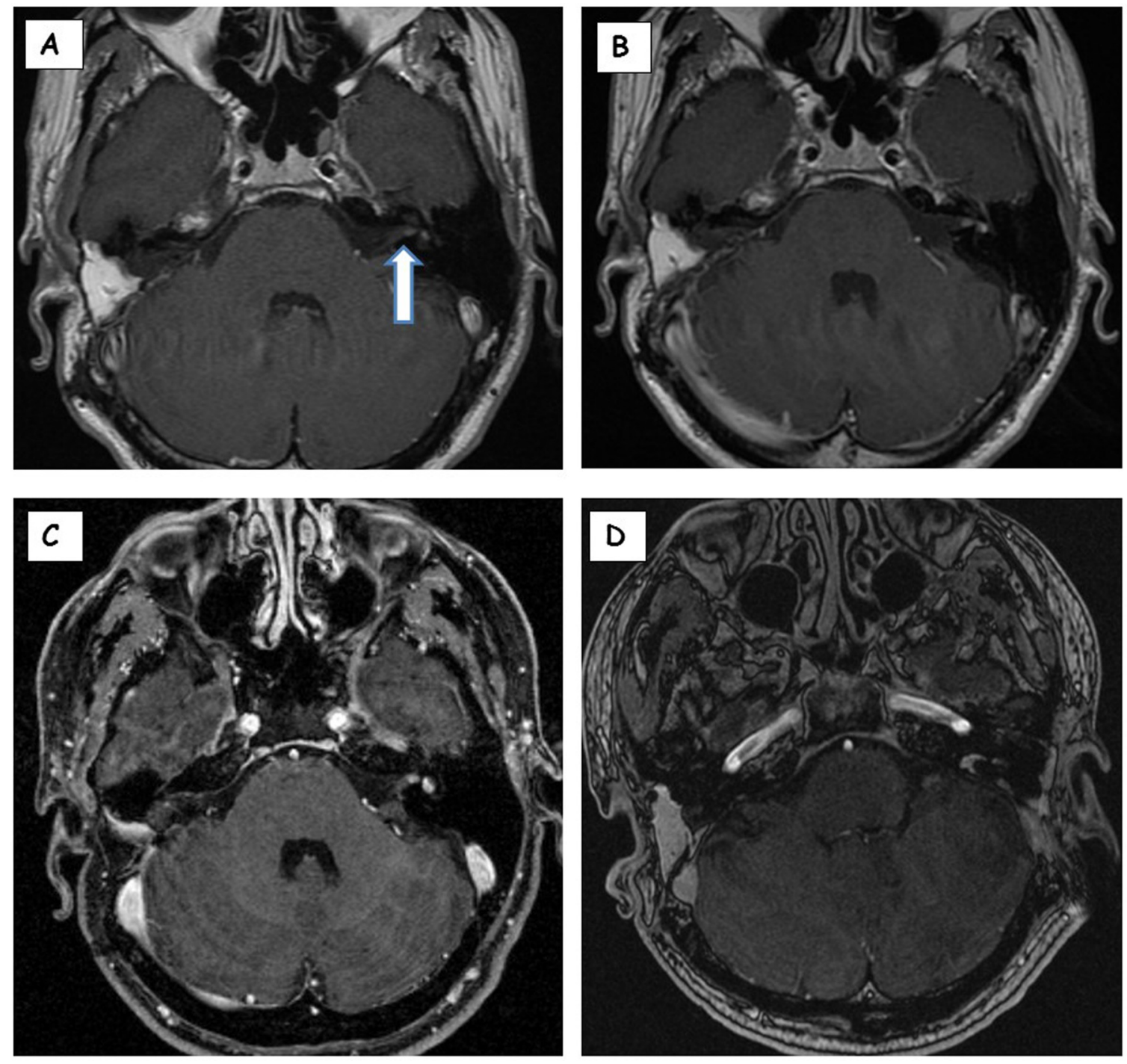

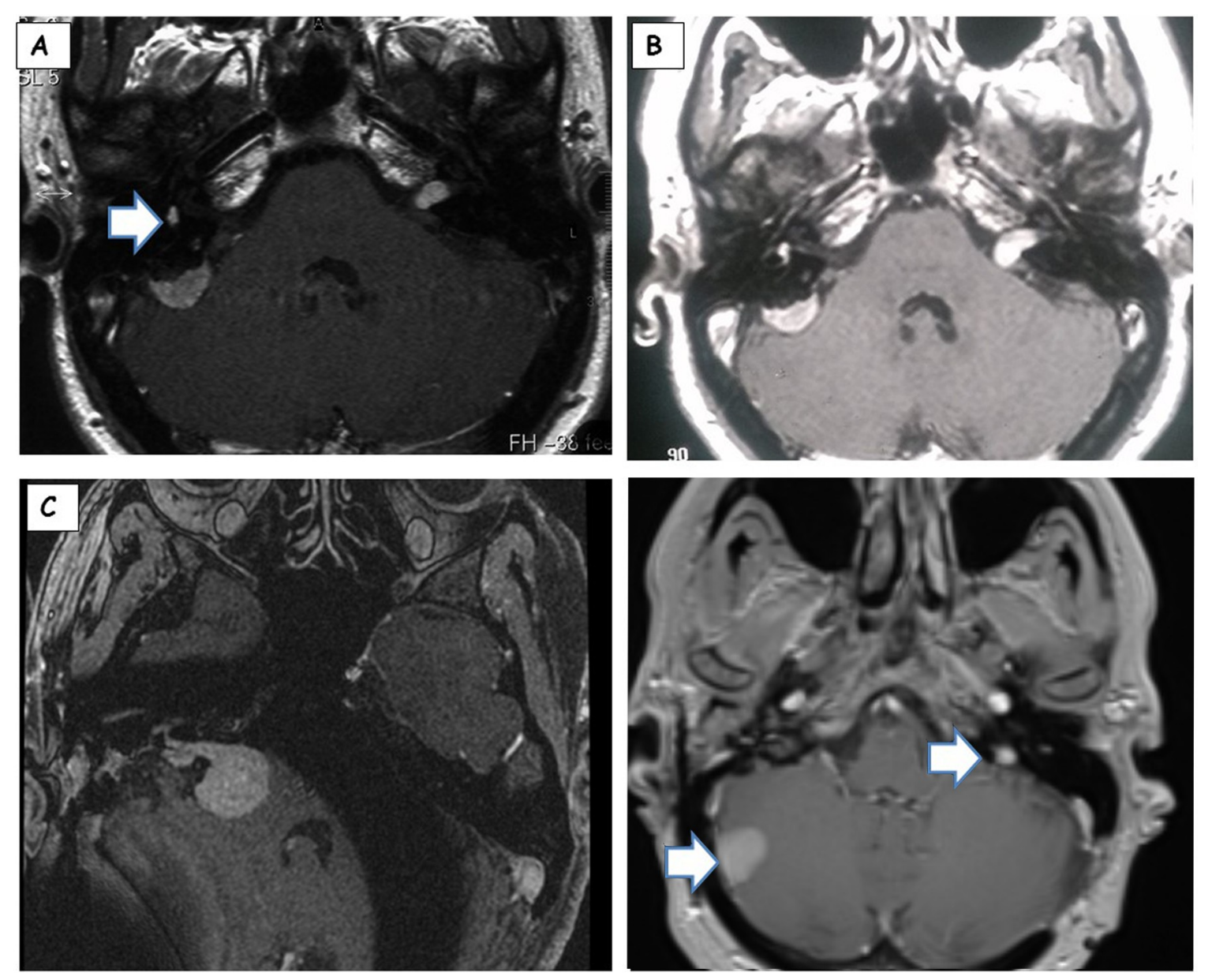